\title{
Electron Cyclotron Emission Simulation from TCABR Plasmas
}

\author{
Eduardo H. Lyvio and P. R. da S. Rosa \\ Departamento de Física, UFMS, Caixa Postal 549, 79070-900, Campo Grande, MS, Brazil
}

Received on 26 January, 2004; revised version received on 29 April, 2004

\begin{abstract}
Electron cyclotron emission due to electrons described by a particular distribution function has been studied. The latter presents an extended tail generated by the interaction of the Lower Hybrid wave with the plasma as compared to the Maxwellian distribution function. For this purpose a new code has been developed which calculates for an arbitrary distribution function the intensity of radiation arriving at the plasma edge, the emission profile (as a function of position) and the optical depth (as a function of frequency) using the full dielectric tensor for a magnetized plasma. The electron distribution function is obtained by solving the Fokker-Planck equation in the frame of the quasilinear theory using a slab model. Results obtained for TCABR-like parameters show changes in the emission localized at positions where electron distribution function has been modified by the waves. Main parameter governing the changes in the electron cyclotron emission is the wave power. Changes in the plasma temperature and density profiles do not alter the emission profiles substantially. Reconstructed electron temperature profile has been obtained from the code radiation emission simulation, showing good agreement with the imposed temperature profile. The present results also showed that the changes in the emission profile in the region where the Lower Hybrid wave deposes its energy as compared with the emission profile of the plasma with Maxwellian distribution function are not so strong.
\end{abstract}

\section{Introduction}

Radiation emitted by electrons gyrating around the magnetic field lines (ECE) at the electron cyclotron frequency and its harmonics is an important diagnostic tool in plasmas confined magnetically, mainly to determine the plasma temperature profile. This method takes advantage of spatial dependence of the cyclotron frequency in a tokamak plasma which is determined mainly by the dependence of the toroidal magnetic field on position. For tokamaks the toroidal magnetic field decays inversely with the major radius $\left(B_{0} \propto \frac{1}{R}\right)$. For such devices the frequency of the emitted radiation can be related directly to spacial position [2]. This hypothesis is actually true only for Maxwellian plasmas. When there is a non-Maxwellian distribution function we have to investigate if we can still relate the emission observed at plasma edge in a given frequency with only one position inside the plasma.

This paper is dedicated to the study of the intensity of ECE at the plasma edge as changed by the presence of the Lower Hybrid waves ( $\mathrm{LH})$ in comparison with the case in which the electron distribution function is the Maxwellian one. In contrast to a previous work on this subject [3], where cold expressions for both the dielectric tensor and dispersion relation were used for the evaluation of the emission coefficient, we make use of the hot expressions for these quantities. LH waves are purely electrostatic modes and can propagate into the plasma to interact with electrons through Landau damp mechanism, transferring parallel momentum to the electrons belonging to the electron distribution tail. Throughout this paper the expressions parallel and perpendicular refer, respectively, to the $\mathbf{B}_{\mathbf{0}}$ direction $\left(\mathbf{B}_{\mathbf{0}}=\mathbf{B}_{\mathbf{0}} \mathbf{e}_{\mathbf{z}}\right)$ and the direction perpendicular to it.
The paper is organized as follows. Section 2 discusses the equations which are employed and the approximations made. Section 3 shows the results for ECE found by numerical solution of the Fokker-Planck and energy transport equations, as a function of several parameters: LH injected power, plasma temperature and plasma density. Section 4 presents our main conclusions.

\section{Numerical scheme and plasma mo- del}

For the calculation of the emission coefficient of EC waves we need to know the dielectric tensor $\overleftrightarrow{\varepsilon}$ and the microscopic current correlation tensor $\overleftrightarrow{G}$. For the case of wave emission perpendicular to the toroidal magnetic field considered in this paper, the emission coefficient takes a very simple form, since tensor components $\varepsilon_{13}, \varepsilon_{23}, G_{13}$ and $G_{23}$ are negligible. Thus, the emission coefficient for the extraordinary mode is given by $[3,5]$

$$
\beta_{x}=\frac{4 \pi^{2} \omega^{2}}{c^{3} n}\left|1-\frac{i \varepsilon_{12}}{\varepsilon_{11}}\right|^{2} G_{11}
$$

where $\omega$ is the wave frequency; $n$ is the refraction index for the extraordinary mode (here taken as being the real part of the complex refractive index, evaluated by solving the appropriate dispersion relation); $\varepsilon_{i j}$ are the components of the hot dielectric tensor and $G_{11}$ is given by 


$$
\begin{aligned}
G_{11}= & \frac{1}{\nu_{e 0}^{l-2}} \frac{\omega T_{e}}{(2 \pi)^{4}} \frac{\pi X}{2^{2 l}[(l-1) !]^{2}} \times \\
& {\left[\frac{n}{y_{1}}\right]^{2(l-1)} u_{*}^{2 l+1} \int_{-1}^{1} d \mu\left(1-\mu^{2}\right) f_{*} . }
\end{aligned}
$$

The quantities appearing in the equation 1 are $f(u, \mu, x, \tau)$, the electron distribution function ( $\tau$ is the time normalized to the collision time at the center of the slab); $u$ is the electron momentum normalized to the central thermal momentum at $\tau=0 ; \mu=\cos \theta$ is the cosine of the pitch angle $\theta ; \gamma \equiv\left[1+\frac{u^{2}}{\nu_{e 0}}\right]^{1 / 2}$ is the relativistic factor $\left(\nu_{e 0}=m c^{2} / T_{e 0}\right) ; \omega_{c}$ the electron cyclotron frequency, $l$ is the harmonic number, $X \equiv \omega_{p}^{2} / \omega^{2}$ (where $\omega_{p}$ is the electron plasma frequency); $T_{e 0}$ is the central plasma temperature (in $\mathrm{keV}) ; u_{*}=\left[\nu_{e 0}\left(y_{l}^{2}-1\right)\right]^{1 / 2}$ is the resonating speed of electrons that can emit EC waves at frequency $\omega$ and $y_{l}=l \omega_{c} / \omega$.

The dielectric tensor can be written as

$$
\overleftrightarrow{\varepsilon}=\overleftrightarrow{\varepsilon}+i \overleftrightarrow{\varepsilon} \prime \prime
$$

where the first and second terms in the right hand side denote, respectively, the Hermitian and anti-Hermitian parts of the hot dielectric tensor. For the evaluation of the Hermitian part we make use of a relativistic Maxwellian distribution function while for the evaluation of the anti-Hermitian part, considering a harmonic number $l$, we make use of the following expression (valid for $n_{\|}=0$ )

$$
\begin{aligned}
\varepsilon_{i j, l}^{\prime}= & -2 \pi^{2} l^{2} \frac{X \nu_{e 0} \gamma_{*}}{u_{*}} \int_{-1}^{1} d \mu\left(1-\mu^{2}\right) \\
& \times\left[\frac{u^{2}}{\rho^{2}} \Pi_{i l}^{*} \Pi_{j l}\left(\mu \partial_{u}-\mu \partial_{\mu}\right) f\right]_{u=u_{*}} .
\end{aligned}
$$

Here $\rho=\frac{n_{\perp} u_{\perp}}{y_{1} \nu_{e 0}^{1 / 2}}$, where $n_{\perp}$ is the real part of the perpendicular component of the complex refractive index $\mathbf{n}$. Other quantities appearing in the equation 2 are

$$
\begin{aligned}
\Pi_{1, l} & =J_{l}(\rho)=\left(\frac{1}{2}\right)^{l} \frac{\rho^{l}}{l !}, \\
\Pi_{2, l} & =-i \frac{\rho J_{l}(\rho)}{l}=-i \Pi_{1, l} \\
\Pi_{3, l} & =\frac{n_{\perp}}{y_{l}} \frac{\mu u_{*}}{\nu_{e 0}^{1 / 2}} J_{l}(\rho)=\frac{n_{\perp}}{y_{l}} \frac{\mu u_{*}}{\nu_{e 0}^{1 / 2}} \Pi_{1, l}
\end{aligned}
$$

These expressions are valid in the small Larmor radius approximation. By making use of these expressions the emission coefficient for perpendicular propagation to the toroidal magnetic field for the extraordinary mode can be written in the following way

$$
\begin{aligned}
\beta_{x}= & \frac{1}{2^{2 l+2}}\left(\frac{\omega}{c}\right)^{3} \frac{X T_{e}}{\pi n \nu_{e 0}^{l-2}} \frac{u_{*}^{2 l+1}}{[(l-1) !]^{2}}\left[\frac{n}{y_{1}}\right]^{2(l-1)} \\
& \times\left|1-\frac{i \varepsilon_{12}}{\varepsilon_{11}}\right|^{2} \int_{-1}^{1} d \mu\left(1-\mu^{2}\right)^{l} f_{*}
\end{aligned}
$$

In order to simulate the tokamak plasma we make use of a semi-infinite slab model as shown in Fig. 1.
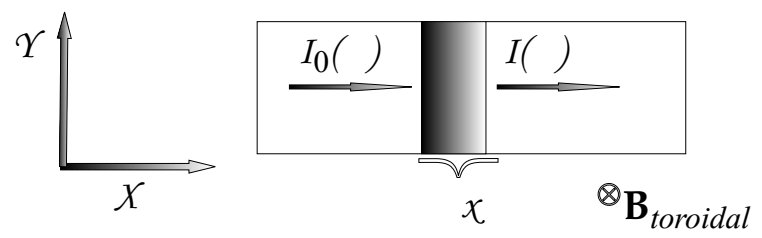

Figure 1. Slab model.

We consider the slab having width $\triangle x$ in the $x$ direction and infinite in $z$ direction (magnetic field direction). To calculate the spectral intensity at the plasma edge the slab is divided into small pieces each one having width $\triangle x_{j}$ in the $x$ direction ([1]). Within such small pieces all parameters are assumed to be constant. For a given frequency the transport equation gives the spectral intensity emitted by the plasma at each small slab border. Using the values of the emitted spectral intensity $\left(\beta_{x}\right)$ and the absorption coefficient for each small slab $(\alpha)$, the cumulative spectral intensity of the radiation transmitted through the plasma $(I(\omega))$ can be calculated by $\left(n_{r}\right.$ being the real part of the refractive index)

$$
I(\omega)=I_{0}(\omega) e^{-\alpha \Delta x}+\frac{\beta_{x}}{\alpha n_{r}^{2}}\left[1-e^{-\alpha \Delta x}\right]
$$

We start with a Maxwellian distribution function and, as the LH waves interact with the plasma, a tail in the electron distribution function is generated. The tail appears as a result of the competition between the diffusion process in the momentum space due to the LH waves and the collisions, until the steady state is reached. All results shown involve numerical simulations for the TCABR Tokamak [4] whose main parameters are: major radius $\left(R_{0}\right) 61.5 \mathrm{~cm}$; minor radius (a) $18.0 \mathrm{~cm}$; central toroidal magnetic induction $\left(\mathbf{B}_{\mathbf{0}}\right.$ ) 12000 Gauss, central electron density $\left(n_{e 0}\right) 1.5$ up to 2.0 $\times 10^{13} \mathrm{~cm}^{-3}$ and central electron temperature $\left(T_{e 0}\right) 0.5$ up to $1.5 \mathrm{keV}$. Density and temperature profiles are given by

$$
\begin{aligned}
& n_{e}=\left(n_{e 0}-n_{e a}\right)\left(1-\frac{x^{2}}{a^{2}}\right)+n_{e a} \\
& T_{e}=\left(T_{e 0}-T_{e a}\right)\left[1-\frac{x^{2}}{a^{2}}\right]^{2}+T_{e a} .
\end{aligned}
$$

In these expressions $n_{e a}$ is the edge electron density (1.5 up to the $\left.2.0 \times 10^{11} \mathrm{~cm}^{-3}\right)$ and $T_{e a}$ is the edge electron temperature $\left(0.5 \times 10^{-2}\right.$ up to $\left.1.5 \times 10^{-2} \mathrm{keV}\right)$. Fig. 2 shows both profiles for a particular value of the central temperature and central density. 


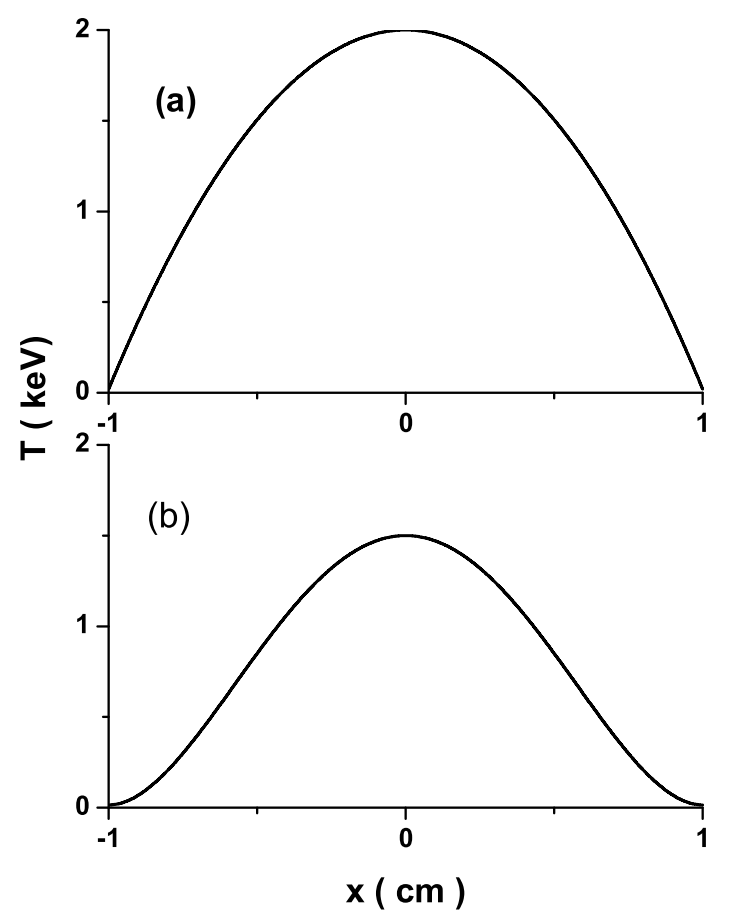

Figure 2. $a$ Plasma Density profile; $b$ Plasma temperature profile.

The LH wave power runs from 0.1 up to $0.3 \mathrm{MW}$. For the parameters chosen the $\mathrm{LH}$ waves will resonate with electrons belonging to the tail of the electron distribution in the region $2.5<u_{\|}<5.5$.

To evaluate the spectral intensity arriving at the plasma edge we need to calculate the absorption coefficient $(\alpha)$, the real part of the complex refraction index $\left(n_{r}\right)$ and the emission coefficient $\left(\beta_{x}\right)$. For this purpose a new code called Emissao has been developed that will supply us with these quantities. The algorithm we use is the following: we start with a Maxwellian distribution function which is altered by the LH waves until a steady state is reached. The distribution function is the output of the code called kinesis [6], for several values of the $\mathrm{LH}$ power $\left(P_{l h}\right)$, central temperature $\left(T_{e 0}\right)$ and central density $\left(n_{e 0}\right)$. The previously calculated distribution function is then used as the input of the code Emissao. This code calculates the Hermitian and anti-Hermitian components of the dielectric tensor, which will be used in the dispersion relation that provides the refraction index. The refraction index is then used for the calculation of the absorption coefficient.

In order to solve this problem we discretize the threedimensional $(u, \mu, x)$ space by making use of a grid of $151 \times 71 \times 501$ points and use the ADI method to solve the resultant system of equations. With these parameters the code runs comfortably in a PC with a Pentium $1.5 \mathrm{GHz}$ processor and 512 Mbytes of the RAMBUS PC-800 memory kind.

\section{Numerical results}

We start by investigating the dynamical behavior of the $\mathrm{LH}$ power deposition profile. In order to understand this beha- vior we show in Fig. 3 the LH power deposition profile $P_{l h}$, the total LH power absorbed between two neighboring surfaces located at $r$ and $r+d r$, given by

$$
P_{l h}(r, r+d r)=4 \pi^{2} R_{0} \int_{r}^{d r} d r^{\prime} r^{\prime} \rho_{l h}\left(r^{\prime}, \tau\right)
$$

and

$$
\rho_{l h}=1.5 x 10^{-16} T_{e 0} \nu_{e 0} \int d^{3} u \frac{u^{2}}{2}\left(\partial_{\tau} f\right)_{L H}
$$

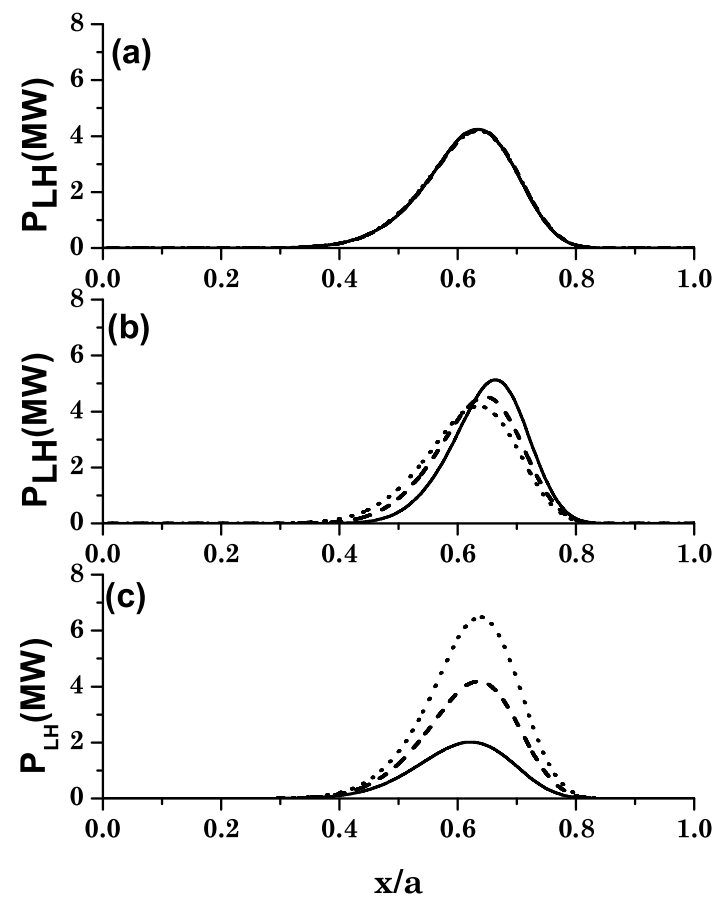

Figure 3. Power absorbed profile: $(a) T_{e 0}=1.5 \mathrm{keV}, P_{l h}=0.2$ MW and $n_{e 0}=1.5 \times 10^{13} \mathrm{~cm}^{-3}$ (solid line), $1.75 \times 10^{13} \mathrm{~cm}^{-3}$ (dashed line) and $2.0 \times 10^{13} \mathrm{~cm}^{-3}$ (dotted line); $(b) P_{l h}=0.2$ MW, $n_{e 0}=2.0 \times 10^{13} \mathrm{~cm}^{-3}$ and $T_{e 0}=0.5$ (solid line), 1.0 (dashed line) and 1.5 (dotted line); $(c) T_{e 0}=1.5 \mathrm{keV}, n_{e 0}=$ $2.0 \times 10^{13} \mathrm{~cm}^{-3}$ and $P_{l h}=0.1$ (solid line), 0.2 (dashed line) and 0.3 (dotted line).

Figure 3 shows the dependence of the LH power deposition profile on the electron density (panel $a$ ), electron temperature (panel $b$ ) and LH power at the plasma edge (panel $c$ ). From the first panel we can see that, in the range of density values analyzed, the plasma density does not play any important role in the process of LH power absorption. The plasma temperature, however, shows influence on the behavior of the LH power absorption. With the increase of the central temperature the peak of the LH power deposition gradually begins to enlarge and moves toward the center of the plasma. In the panel $(c)$ we can see that the deposition region of LH power remains essentially the same as LH power at plasma edge is increased. 
Since LH waves transfer momentum in the parallel direction it is interesting to investigate the behavior of the parallel distribution function $\left(f_{\|}\right)$, defined by

$$
f_{\|}=2 \pi \int_{0}^{\infty} d u_{\perp} u_{\perp} f\left(u_{\|}, u_{\perp}\right)
$$

where the components of the normalized momentum are $u_{\|}=u \mu$ and $u_{\perp}=u\left(1-\mu^{2}\right)^{1 / 2}$. Fig. 4 shows the behavior of $f_{\|}$for several values of LH power, central temperature and density, at steady state, in the slab position where the maximum LH power deposition is located (between 10 and $12 \mathrm{~cm})$.
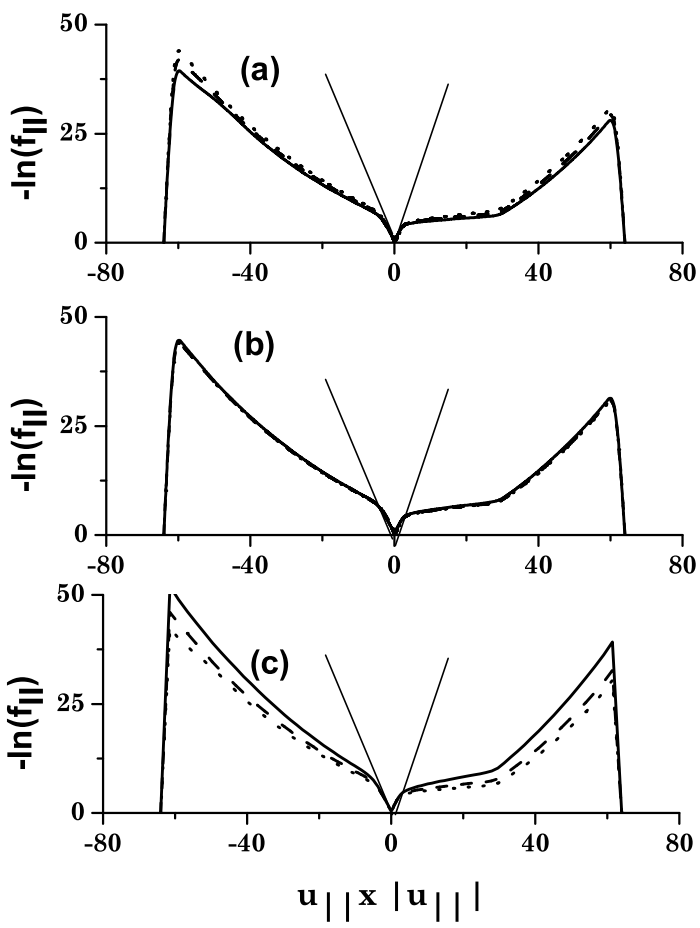

Figure 4. Parallel distribution function. ( $a$ ) $\mathrm{P}_{l h}=0.2 \mathrm{MW}, \mathrm{T}_{e 0}=1.5$ $\mathrm{keV}$ and $\mathrm{n}_{e 0}=1.5 \times 10^{13} \mathrm{~cm}^{-3}$ (full line), $1.75 \times 10^{13} \mathrm{~cm}^{-3}$ (dashed line) and $2.0 \times 10^{13} \mathrm{~cm}^{-3}$ (dotted line); $(b) \mathrm{P}_{l h}=0.2 \mathrm{MW}$, $\mathrm{n}_{e 0}=2.0 \times 10^{13} \mathrm{~cm}^{-3}$ and $\mathrm{T}_{e 0}=0.5 \mathrm{keV}$ (full line), $1.0 \mathrm{keV}$ (dashed line) and $1.5 \mathrm{keV}$ (dotted line); $(c) \mathrm{T}_{e 0}=1.5 \mathrm{keV}, \mathrm{n}_{e 0}=2.0 \times 10^{13}$ $\mathrm{cm}^{-3}$ and $\mathrm{P}_{l h}=0.1 \mathrm{MW}$ (full line), $0.2 \mathrm{MW}$ (dashed line) and 0.3 MW (dotted line).

In the description of $f_{\|}$we make usage of $-\ln \left(f_{\|}\right)$instead of $f_{\|}$as a function of $u_{\|} \times\left|u_{\|}\right|$. All panels of Fig. 4 show the same behavior. Starting with the Maxwellian distribution function, that in the figure has the form of $V$, as the time goes by and the waves interact with the plasma, a tail in the distribution function is observed. Panel $c$ of Fig. 4 shows remarkable changes in the distribution function as LH power is raised with the formation of an extended and flat tail. It is to be noted that the LH power is the principal parameter responsible for the changes in the electron distribution function. The flat tail is formed as a result of the competition between the diffusion processes in momentum space due to LH waves and collisions. The evolution of the perturbation due to the LH wave is compensated by the collisions, until the steady state is reached.
Besides the formation of the tail in the electron distribution function, due to the diffusion of particles to high parallel momentum region in the momentum space, we have also the diffusion of particles to high perpendicular momentum region due to collisions. This diffusion is given in Fig. 5 which exhibits $T_{\perp} / T_{e}$ versus $u_{\|}$, where $T_{\perp}$ is the perpendicular temperature given by

$$
T_{\perp}\left(u_{\|}\right)=\frac{2 \pi T_{e}}{f_{\|}} \int_{0}^{\infty} d u_{\perp} u_{\perp} \frac{u_{\perp}^{2}}{2} f\left(u_{\|}, u_{\perp}\right)
$$

This figure clearly shows that, in the range of values of the central electron temperature studied, the perpendicular temperature is practically not sensible to changes in the temperature and density values at the plasma center. On the other hand we notice the growth of the perpendicular temperature in the position where LH wave is more pronounced as the LH power at plasma edge is increased, as shown in panel $c$ of the same Figure.
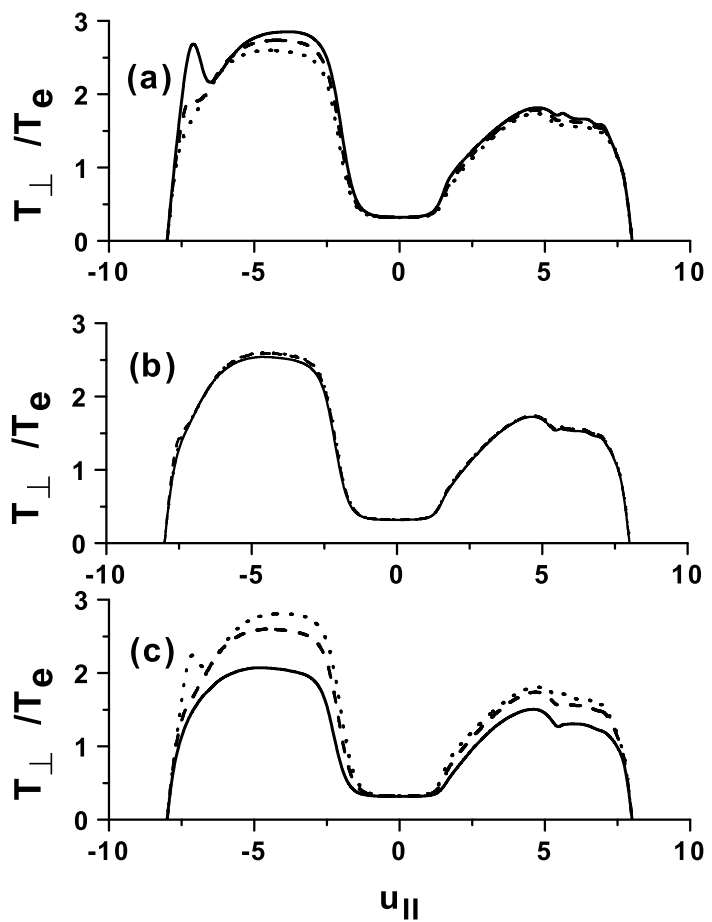

Figure 5. Perpendicular temperature. (a) $\mathrm{P}_{l h}=2.0 \mathrm{MW}, \mathrm{T}_{e 0}=1.5$ $\mathrm{keV}$ and $\mathrm{n}_{e 0}=1.5 \times 10^{13} \mathrm{~cm}^{-3}$ (full line), $1.75 \times 10^{13} \mathrm{~cm}^{-3}$ (dashed line) and $2.0 \times 10^{13} \mathrm{~cm}^{-3}$ (dotted line); (b) $\mathrm{P}_{l h}=0.2 \mathrm{MW}$, $\mathrm{n}_{e 0}=2.0 \times 10^{13} \mathrm{~cm}^{-3} ; \mathrm{T}_{e 0}=0.5 \mathrm{keV}$ (full line), $1.0 \mathrm{keV}$ (dashed line) and $1.5 \mathrm{keV}$ (dotted line); (c) $\mathrm{T}_{e 0}=1.5 \mathrm{keV}, \mathrm{n}_{e 0}=1.5 \times 10^{13}$ $\mathrm{cm}^{-3}$ and $\mathrm{P}_{l h}=0.1 \mathrm{MW}$ (full line), $0.2 \mathrm{MW}$ (dashed line) and 0.3 MW (dotted line).

Figure 6 shows the time evolution of the current driven by the LH waves for several values of the central temperature and density, until the steady state is reached. We can observe that the small difference observed in the flat tail shown in Fig. 4 when integrated results in strong enhancement in 
the current driven except for the dependence of plasma current on density. In this case higher densities mean more collisions and lower efficiency of the current drive process.

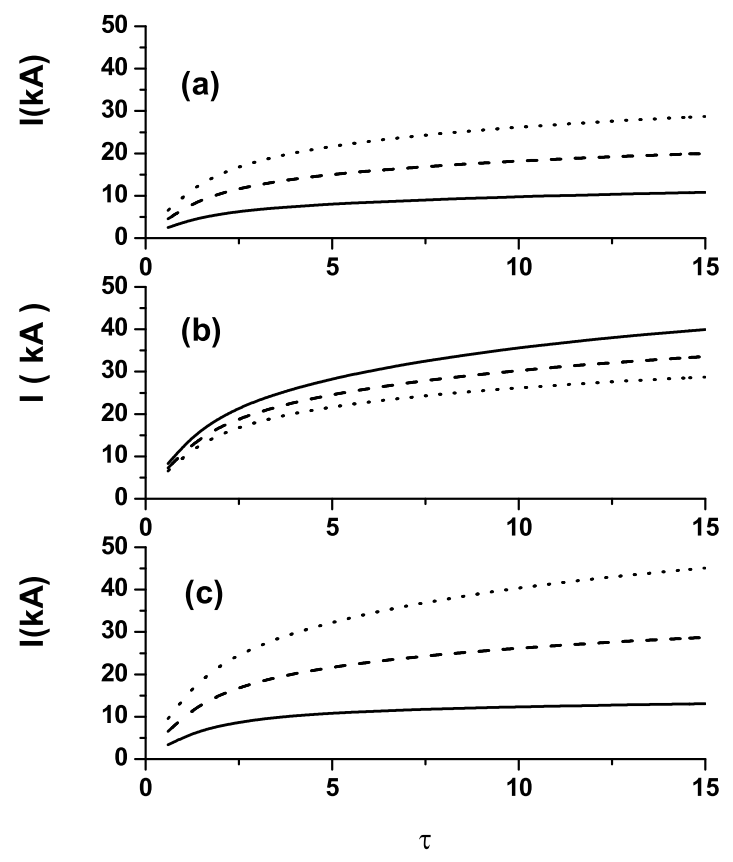

Figure 6. Current driven. (a) $P_{l h}=0.2 \mathrm{MW}, T_{e 0}=1.5 \mathrm{keV}$ and $n_{e 0}=1.5 \times 10^{13} \mathrm{~cm}^{-3}$ (full line), $1.75 \times 10^{13} \mathrm{~cm}^{-3}$ (dashed line) and $2.0 \times 10^{13} \mathrm{~cm}^{-3}$ (dotted line); $(b) P_{l h}=0.2 \mathrm{MW}, n_{e 0}=$ $2.0 \times 10^{13} \mathrm{~cm}^{-3}$ and $T_{e 0}=0.5 \mathrm{keV}$ (full line), $1.0 \mathrm{keV}$ (dashed line) and $1.5 \mathrm{keV}$ (dotted line); $(c) T_{e 0}=1.5 \mathrm{keV}, n_{e 0}=2.0 \times 10^{13}$ $\mathrm{cm}^{-3}$ and $P_{l h}=0.1 \mathrm{MW}$ (full line), 0.2 MW(dashed line), 0.3 MW (dotted line).

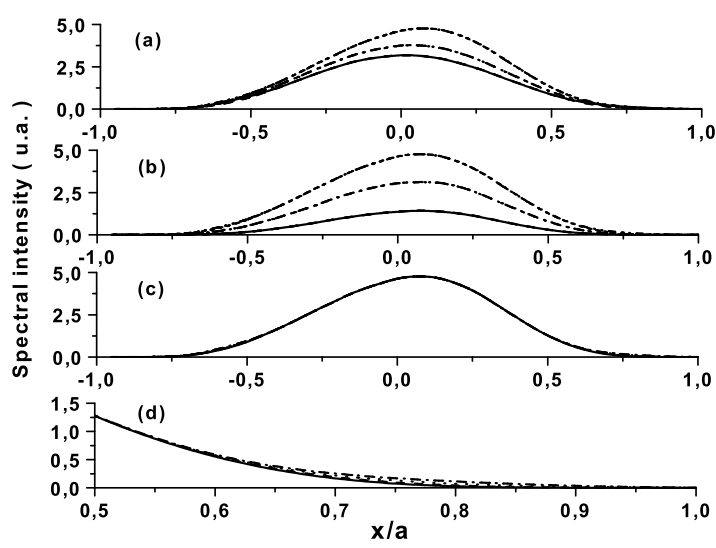

Figure 7. Spectral intensity. (a) $\mathrm{P}_{l h}=0.2 \mathrm{MW}$ and $\mathrm{T}_{e 0}=1.5$ keV. $\mathrm{n}_{e 0}=1.5 \times 10^{13} \mathrm{~cm}^{-3}$ (full line), $1.75 \times 10^{13} \mathrm{~cm}^{-3}$ (dashed line) and $2.0 \times 10^{13} \mathrm{~cm}^{-3}$ (dotted line). (b) $\mathrm{P}_{l h}=2.0 \mathrm{MW}$ and ne $0=2.0 \times 10^{13} \mathrm{~cm}^{-3} ; \mathrm{T}_{e 0}=0.5 \mathrm{keV}$ (full line), $1.0 \mathrm{keV}$ (dashed line) and $1.5 \mathrm{keV}$ (dotted line). (c) $\mathrm{T}_{e 0}=1.5 \mathrm{keV}$ and $\mathrm{n}_{e 0}=2.0 \times 10^{13}$ $\mathrm{cm}^{-3}$. $\mathrm{P}_{l h}=0.1 \mathrm{MW}$ (dashed line), 0.2 MW (dotted line), 0.3 MW (dashed-dotted line); (d) Amplification of the curves shown in panel $c$. The corresponding values for Maxwellian distribution function overcome those of perturbed distribution function in all graphs.
We now proceed to analyze the intensity of ECE, the optical depth and the emission coefficient which were found taken into account the plasma dielectric properties. Fig. 7 shows the spectral intensity as a function of plasma density (panel $a$ ), plasma temperature (panel $b$ ) and LH power at the plasma edge (panel $c$ ). In all panels, the Maxwellian values are hardly visible being superimposed by the values of spectral intensity after LH waves and collision reached the steady state. From panel (c) we observe that LH power level plays a minor role in the ECE at plasma edge. There is only a weak dependence on LH power, shown in panel $d$ of the figure. In this panel we amplify the region where the LH waves depose their power. All cases studied show the same behavior: the spectral intensity values obtained from the Maxwellian distribution function are not quite different from the ones found making use of the perturbed electron distribution function.

Figure 8 shows the dependence of the ECE coefficient on plasma density (panels $a$ and $b$ ), plasma temperature (panels $c$ and $d$ ) and LH power at the plasma edge (panels $e$ and $f$ ). In this Figure the profiles obtained from the Maxwellian distribution function (on the left side) and for the perturbed distribution function (on the right side) are shown for a particular frequency $(55 \mathrm{GHz})$. In all cases is observed the influence of the LH waves on the ECE coefficient. Although the peak values of the ECE coefficient are the same for Maxwellian and perturbed distribution function cases, the curves are wider in the perturbed distribution function cases as compared with the Maxwellian ones. The values of the ECE coefficient are increased in all cases studied with the values obtained for the perturbed distribution function being higher than the Maxwellian ones closer to the plasma center.
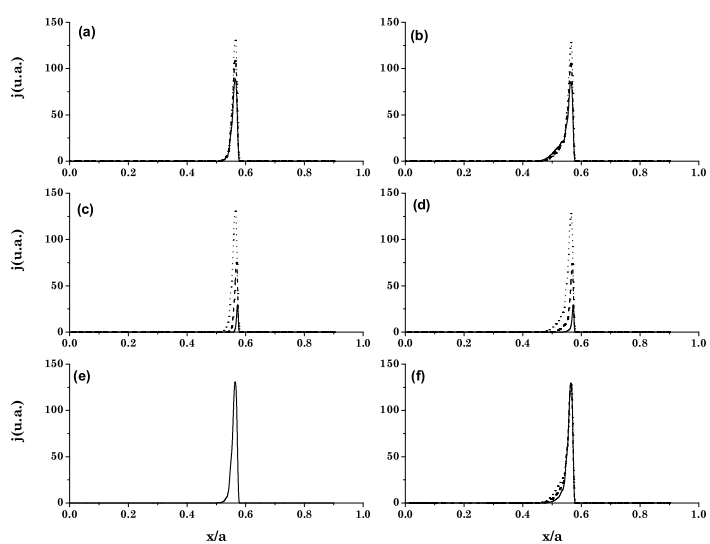

Figure 8. Dependence of ECE coefficient on: electron density [panel (a) Maxwellian function, panel (b) perturbed function: $P_{l h}=0.2 \mathrm{MW}, T_{e 0}=1.5 \mathrm{keV}, n_{e 0}=1.5 \times 10^{13} \mathrm{~cm}^{-3}$ (full line), $n_{e 0}=1.75 \times 10^{13} \mathrm{~cm}^{-3}$ (dashed line), $n_{e 0}=2.0 \times 10^{13}$ $\mathrm{cm}^{-3}$ (dotted line)]; electron temperature [panel (c) Maxwellian function, panel (d) perturbed function: $P_{l h}=0.2 \mathrm{MW}, n_{e 0}=$ $2.0 \times 10^{13} \mathrm{~cm}^{-3}, T_{e 0}=0.5 \mathrm{keV}$ (full line), $T_{e 0}=1.0 \mathrm{keV}$ (dashed line), $T_{e 0}=1.5 \mathrm{keV}$ (dotted line)]; and LH power [ panel (e) Maxwellian function, panel (f) perturbed function: $T_{e 0}=1.5$ $\mathrm{keV}, n_{e 0}=2.0 \times 10^{13} \mathrm{~cm}^{-3}, P_{l h}=0.1 \mathrm{MW}$ (full line), $P_{l h}=0.2$ $\mathrm{MW}$ (dashed line), $P_{l h}=0.3 \mathrm{MW}($ dotted line $\left.)\right]$. 
The optical depth $\left(\tau_{x}\right)$ dependence on plasma density (panel $a$ ), plasma temperature (panel $b$ ) and $\mathrm{LH}$ power at the plasma edge (panel $c$ ) are shown in Fig. 9. As before we can not observe any difference between the Maxwellian case and the perturbed one in panels $a$ and $b$. As the power is increased (panel $c$ ) little modifications can be observed in the optical depth profile in the region where the LH waves deposit their power. These changes may be better appreciated in panel $d$, where an amplification of this region is shown.
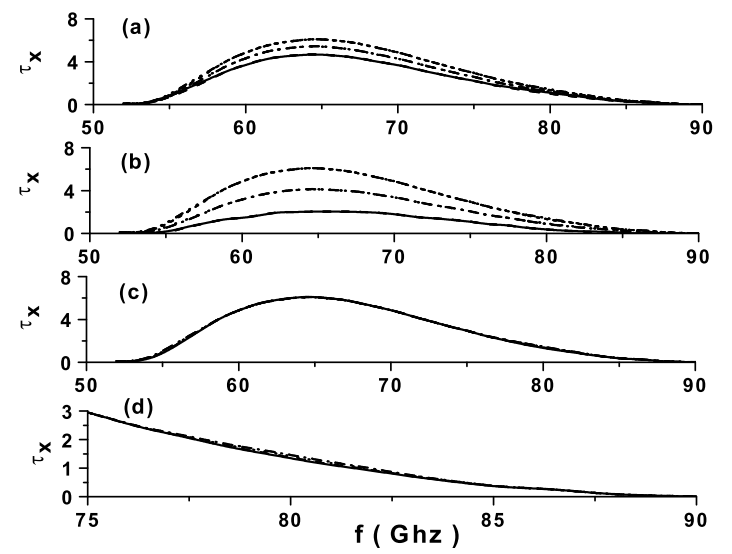

Figure 9. Optical Depth. (a) $P_{l h}=0.2 \mathrm{MW}, T_{e 0}=1.5 \mathrm{keV}$ and $n_{e 0}=1.5 \times 10^{13} \mathrm{~cm}^{-3}$ (full line), $1.75 \times 10^{13} \mathrm{~cm}^{-3}$ (dashed line) and $2.0 \times 10^{13} \mathrm{~cm}^{-3}$ (dotted line). $(b) P_{l h}=0.2 \mathrm{MW}, n_{e 0}=2.0 \times$ $10^{13} \mathrm{~cm}^{-3}$ and $T_{e 0}=0.5 \mathrm{keV}$ (full line), $1.0 \mathrm{keV}$ (dashed line) and $1.5 \mathrm{keV}$ (dotted line). (c) $T_{e 0}=1.5 \mathrm{keV}, n_{e 0}=2.0 \times 10^{13} \mathrm{~cm}^{-3}$ and $P_{l h}=0.1 \mathrm{MW}$ (dashed line), $0.2 \mathrm{MW}$ (dotted line), 0.3 MW (dashed dotted line) and Mawellian (full line). (d) Amplification of the region on panel $c$ where LH waves deposit their power.

Although the LH power is deposited on the low field side of the tokamak, we can also notice changes in the optical depth localized on the high field side. These changes arise because the electrons receiving energy on the low field side follow the helicoidal lines of the magnetic field modifying the distribution function on the high field side.

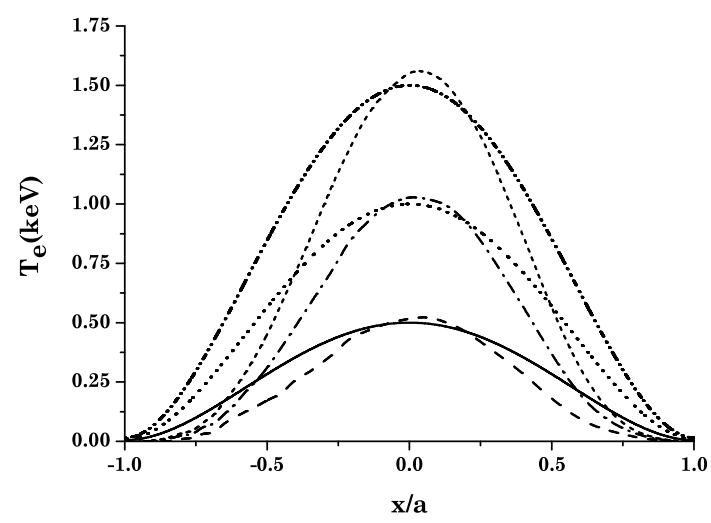

Figure 10. Plasma temperature profile. $\mathrm{T}_{e 0}=0.5 \mathrm{keV}[\operatorname{Imposed}(\mathrm{full}$ line), reconstructed(dashed line)], $1.0 \mathrm{keV}$ [Imposed(dotted line), reconstructed(dashed-dotted line)] and $1.5 \mathrm{keV}$ [Imposed(dashed dotted dotted line) and reconstructed(short dashed line)].
Figure 10 shows a comparison among the imposed and reconstructed temperature profiles for Maxwellian plasmas. These curves were obtained from the calculated emission values for the following values of $T_{e 0}: 0.5,1.0$ and $1.5 \mathrm{keV}$. The expression for the radiation temperature is given by

$$
T_{e}=\frac{8 \pi^{3}}{\omega^{2}} \frac{n_{r}^{2} c^{2}}{\left(1-e^{-\tau_{X}}\right)} I(\omega)
$$

where $\tau_{X}$ is the optical depth for the extraordinary mode.

We observe a good agreement between the temperature profile imposed by equation 5 at positions near the plasma center. This agreement is not so good for positions at middle way between the plasma center and the plasma edge. At these positions the calculated values are always lower than the imposed ones.

\section{Conclusion}

In this work we have analyzed how the presence of a superthermal tail in the electron distribution function, generated by the interaction of LH waves with electrons, influences the ECE at second harmonic of the extraordinary mode.

The results obtained from the numerical solution of the Fokker-Planck and energy transport equations applied to the TCABR parameters show that there are only small differences in the ECE between a plasma with a Maxwellian distribution function and plasmas with a distribution function with the superthermal tail. The importance of these results comes from the assumption that we can use the ECE as a diagnostic tool to measure plasma temperature. This assumption is actually true only for Maxwellian plasmas, which present a very localized region where the emission at a given frequency may occur. Our results show that for TCABR plasmas with a tail in the electron distribution function the method still gives the plasma temperature profile with reasonable accuracy.

This work has been partially supported by Universidade Federal de Mato Grosso do Sul (UFMS), Fundação Coordenação de Aperfeiçoamento de Pessoal de Nível Superior (CAPES), Fundação de Apoio ao Desenvolvimento do Ensino, Ciência e Tecnologia do Estado de Mato Grosso do Sul (FUNDECT).

One of us (PRSR) would like to thanks Dr. Ruy Pepe da Silva (IF-USP) for very useful discussions when developing of the code Emissao.

\section{References}

[1] C. M. Celata and D. A. Boyd. Nuclear Fusion 17, 735 (1979).

[2] F. Engelmann and M. Curatolo. Nuclear Fusion 13, 497 (1973).

[3] G. Giruzzi. Nuclear Fusion 28, (1988).

[4] I. Nascimento et al.. IAEA Technical Committee Meeting on Research Using Small Fusion Devices, China,(1999).

[5] L. F. Ziebell and D. Dillenburg. Phys. Fluids 26, 80 (1983).

[6] P. R. da S. Rosa and L. F. Ziebell. Plasma Phys. Contr. Fusion 44, 2065 (2002). 